\title{
Connecting concepts to problem-solving
}

\author{
Stephen Kanim
}

Department of Physics, New Mexico State University, Las Cruces, New Mexico 88003

Traditional quantitative problems of the type commonly found at the end of chapters in physics textbooks are assigned to students most introductory physics courses. Many students use a formula-driven approach to solve these problems that does not rely on understanding underlying physics concepts and that does little to encourage the problem-solving skills employed by experts. In another paper presented at this conference, we gave an example from electric circuits to illustrate the use of "bridging exercises" as part of students' homework to encourage students to solve problems by starting with developed physics concepts and models. ${ }^{1}$ In this paper, we describe our attempts to use the same approach in the context of electrostatics.

\section{Previous Research}

Much of what we know about student problem solving in physics is a result of 'expert/novice' studies, in which the problem solving strategies of experts (typically university physics faculty members) are compared to those of novices (typically students in introductory physics courses). ${ }^{2}$ Researchers have found important differences:

- Experts arrange their knowledge in a hierarchical fashion with more general ideas related to progressively more specific pieces of information. Novices have less knowledge and their knowledge tends to be sparsely connected and poorly organized.

- Experts tend to classify physics problems based on underlying concepts. In contrast, novices tend to classify problems based on the surface features of the problem.

- Experts use multiple representations to clarify a problem. Mathematical solution typically follows a restatement of the problem based on general principles. Novices often start with an equation, substituting given values and then looking for additional equations to give other values. Many students see the concepts as offering at best some guidelines for selection of equations.

Results reported from the Maryland Physics Expectations Survey of students enrolled in introductory physics courses are also pertinent to understanding student approaches to problem solving. ${ }^{3}$ After completing an introductory mechanics course, only about $50 \%$ of the students who completed an introductory mechanics course at a large public university agreed with the statement "When I solve most exam or homework problems, I explicitly think about the concepts that underlie the problem." Many students do not seem to make a strong connection between the concepts and the problems that are part of introductory physics courses.

Effect of conceptual development on problem solving -- electric circuits

Does an increased emphasis on the development of concepts in the introductory course promote more expert-like problem solving? In the context of electric circuits, we found that students often did not apply learned concepts to quantitative problems. We designed homework worksheets that included "bridging exercises" intended to form a more explicit link between 
concepts developed in tutorials and endof chapter problems. After completing the worksheets, more students were able to solve quantitative problems related to current in electric circuits. ${ }^{1}$

\section{Bridging exercises for other topics}

Encouraged by our experience with electric circuits, we wrote worksheets for other topics in the calculus-based electricity and magnetism course. These worksheets also attempted to guide students in their consideration of the concepts underlying more traditional problems. For many of these topics, however, comparatively little research into specific student difficulties existed upon which to base the worksheets. We tried to anticipate student's difficulties with the concepts and with the mathematical steps required to solve commonly assigned quantitative problems, and to encourage solution of these problems without resorting to formula-driven approaches.

We offered optional problem-solving sessions for students in sections in which the worksheets were first assigned. Students worked through the assignments in small groups, with instructors available to answer questions. Our observations of these small groups led us to realize that for many topics the worksheets were poorly matched to students' needs.

As an example, we discuss our experience with a worksheet intended to guide students through a standard problem in electrostatics shown in Figure 1. The steps required to solve this problem include breaking the rod up into small elements of length $d x$, finding the electric field due to one of these charge elements, calculating the component of this field perpendicular to the rod, and then integrating to find the total electric field.

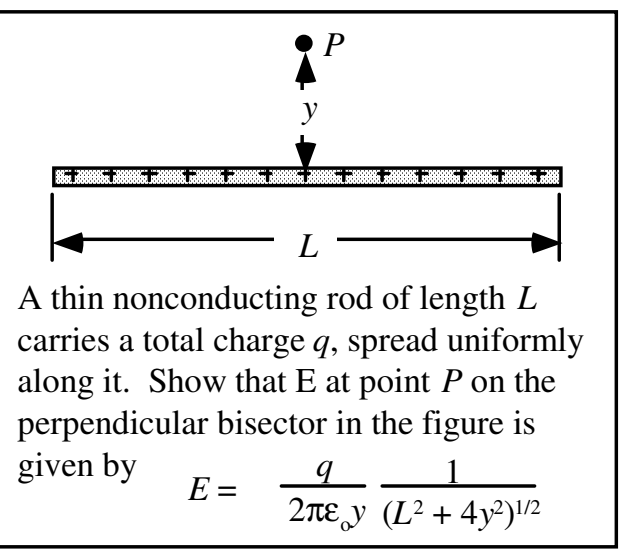

Figure 1. Electric field problem.

The initial steps of the homework worksheet are shown in Figure 2. These questions were intended to allow students to reason qualitatively about factors that determine the electric field.

Criticize the following statement:

"From Coulomb's law, we would expect a force on a positive charge $q_{t}$ placed at point $P$ to have a magnitude

$$
F=\frac{1}{4 \pi \varepsilon_{\mathrm{o}}} \frac{q_{1} q_{2}}{r^{2}}=\frac{1}{4 \pi \varepsilon_{\mathrm{o}}} \frac{q_{1} q_{\mathrm{t}}}{y^{2}} .
$$

So the electric field at point $P$ is the force divided by the test charge:

$$
E=\frac{F}{q_{\mathrm{t}}}=\frac{1}{4 \pi \varepsilon_{\mathrm{o}}} \frac{q}{y^{2}} . "
$$

Would you expect the electric field at point $P$ to be larger or smaller than the value that would be obtained using the method above?

Figure 2. Initial questions for electrostatics worksheet.

We expected that these questions would be relatively easy for students (especially compared to the more mathematical steps that would follow). Most of the students in the optional problem-solving session, however, were unable to answer these questions even after significant guidance. It became clear that we did not have an adequate understanding of student's conceptual difficulties in electrostatics to allow us 
to design effective "bridging" worksheets. We decided that if we hoped to improve students' ability to solve quantitative problems in electrostatics in a more expert-like manner we would first need to understand more about the nature of the underlying conceptual difficulties.

\section{Conceptual difficulties in electrostatics}

We found that for electrostatics, many difficulties exhibited by students were difficulties with the addition of vectors. ${ }^{4}$ Many students treated vectors as scalar quantities when they were asked to add them. Other students were able to show graphically what the sum of vectors would be, but then gave algebraic answers that were inconsistent with their drawings but were consistent with treating the vectors as scalars. Still other students added only the component of a vector that was collinear with the other vector in a vector sum, neglecting the effect of perpendicular components. Additional difficulties with the relationship between vectors and their components were elicited by some of the questions that we asked as part of this study. Many of these difficulties with vector addition were also elicited in topics other than electrostatics. For example, after noticing that some students claimed that vectors of equal magnitude representing electrostatic forces or fields completely cancelled one another (in cases where they were pointing at an acute angle to one another), we asked a similar question in the context of momentum in a mechanics class. This same difficulty was exhibited by about the same proportion of students.

We also discovered difficulties that were specific to electrostatics. For example, many students answer questions in a manner that is consistent with a belief that electric fields can be blocked by the presence of nearby conductors or insulators. Some students reason that insulators act to insulate a charge from the effects of an electric field. Other students give similar reasoning about the effect of conductors.

Some of the difficulties revealed by our investigation were difficulties with fundamental underlying concepts. For example, many students had difficulty differentiating between charge and charge density. We asked students the questions shown in Figure 3 after the completion of instruction in electrostatics up to Gauss' law.

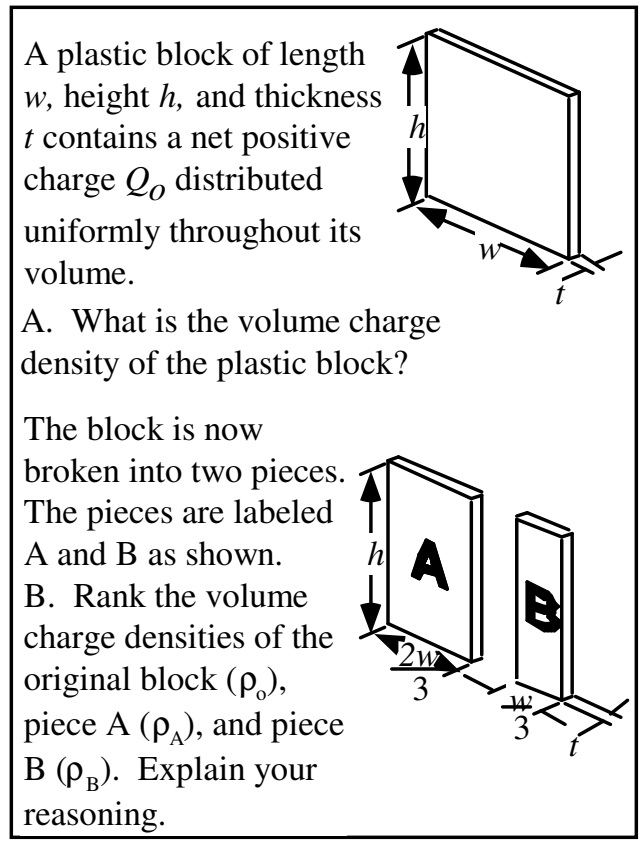

Figure 3. Charged block question.

About $70 \%$ were able to find the charge density from the given information. However, only about 55\% recognized that the charge densities of the pieces would be the same. About $20 \%$ answered that piece B would have the largest charge density. Often student's explanations suggested that they were thinking in terms of the equation for charge density, but were 
holding the charge constant: Similar difficulties with expressions involving more than 2 variables have been noted in different contexts, for example the ideal gas law.

About 20\% answered that the original piece would have the largest charge densities. These responses often included reasoning that suggested a difficulty differentiating between charge and charge density.

Student difficulties with the interpretation of information about charge density were also noted when students were asked to solve problems involving distributed charges. For example, on examination questions where students were expected to use Gauss' law, only about $30 \%$ were able to find the enclosed charge. Many of the incorrect responses included a substitution of the given charge density information into equations that required a charge.

\section{Summary}

Where common conceptual difficulties for a certain topic in physics are well understood, it is possible to design curricula that addresses many of these difficulties. We have found that in these cases an explicit link between the developed concepts and associated quantitative problems ("bridging exercises") increased the number of students who were able to solve quantitative problems. Moreover, the approach taken by students in these cases was often less formula-driven and was more likely to include explicit reference to developed concepts.

In electricity and magnetism, however, we found that often we did not have a sufficient understanding of student's conceptual difficulties to design effective bridging exercises. For many topics, student's exhibited unanticipated difficulties, often with fundamental underlying concepts. If we hope to improve students' ability to solve problems in a more expert-like manner, we need to understand the conceptual issues associated with the problems we are posing, and we need to have developed effective methods for addressing these conceptual issues. Otherwise, student difficulties with problem solving will often reflect conceptual errors with the underlying material.

\section{Acknowledgements}

I would like to thank the many students who participated in the studies described in this paper. The assistance of all members of the Physics Education Group at the University of Washington is gratefully acknowledged, and in particular the substantial contributions made by Peter Shaffer and Lillian McDermott.

\section{References}

${ }^{1}$ S. Kanim, "Connecting concepts about current to quantitative circuit problems," in these conference proceedings.

${ }^{2}$ J. Larkin, J. McDermott, D. Simon, and H. Simon, "Expert and novice performance in solving physics problems," Science 208(20), 1335-1342 (1980).

${ }^{3}$ E. Redish, J. Saul, and R. Steinberg, "Student expectations in introductory physics," American Journal of Physics 66(3), 212-224 (1998).

${ }^{4} \mathrm{~S}$. Kanim, "An investigation of student difficulties in qualitative and quantitative problem solving: Examples from electric circuits and electrostatics," Ph.D. dissertation, Department of Physics, University of Washington (1999). 